\title{
A NOTE ON A FIXED POINT PROPERTY FOR METRIC PROJECTIONS
}

\author{
WAGDY GOMAA EL-SAYED
}

\begin{abstract}
The paper contains a partial answer to a question rasied recently by S.P. Singh concerning the existence of fixed points of metric projections.
\end{abstract}

Let $E$ be a real Banach space with the norm $\|\cdot\|$. Recall that a subset $X$ of $E$ is said to be a Chebyshev if for every $x \in E$ there exists a unique $z \in X$ such that $\|x-z\|=\operatorname{dist}(x, X)$. In this case we can define the so-called metric projection $\mathcal{P}_{X}$ of $E$ onto $X$ which assigns to each $x \in E$ the point $z \in X$ such that $\|x-z\|=\operatorname{dist}(x, X)$.

It is well-known [3] that if $E$ is reflexive and strictly convex then every clsoed convex subset $X$ of $E$ is a Chebyshev set. Thus for every closed convex subset $X$ of a reflexive and strictly convex Banach space $E$ we can define the metric projection $\mathcal{P}_{X}: E \rightarrow X$.

During the conference "Functional Analysis and Applications" held at Gargnano del Garda, Italy (10-14 May 1993) professor S.P. Singh raised the following problem:

Let $A, B$ be Chebyshev sets in a real Banach space $E$ and let $P_{A}, P_{B}$ be the metric projections of $E$ onto the sets $A$ and $B$, respectively. Consider the mapping $P_{A} P_{B}$ : $A \rightarrow A$. Does there exist a fixed point of this mapping?

Observe that in the case when $E$ is a real Hilbert space the answer is affirmative provided $A$ and $B$ are closed, convex and bounded subsets of $E$. It is an easy consequence of the fact that the metric projection is nonexpansive in this setting, so the well-known Browder-Gödhe-Kirk fixed point theorem gives the desired answer (cf. [2]). But it is no longer true for other Banach spaces although they have nice geometrical structure. For example, if $E$ is uniformly convex then the metric projection is only continuous [2].

Nevertheless we show that a large class of Banach spaces has fixed point property with respect to the mapping $P_{A} P_{B}$.

We define

$$
\operatorname{dist}(A, B)=\inf \{\|a-b\|: a \in A, b \in B\} .
$$

Let us start with the following Lemma.

Received June 16, 1994; revised December 8, 1994. 
Lemma 1. Let $A$ and $B$ be Chebyshev sets in an arbitrary Banach space $E$. If there exist points $a \in A$ and $b \in B$ such that $\|a-b\|=\operatorname{dist}(A, B)$ then $a$ is the fixed point of the mapping $P_{A} P_{B}$.

The proof is trivial and is therefore omitted.

Our main result answering the question of S.P. Singh for a large class of Banach spaces is contained in the following theorem.

Theorem 1. Let $E$ be a reflexive and strictly convex Banach space and let $A, B$ be nonempty, bounded, closed and convex subsets of $E$. Then there exist points $a \in A$ and $b \in B$ such that $\|a-b\|=\operatorname{dist}(A, B)$ and simultaneously $a$ is the fixed point of the mapping $P_{A} P_{B}$.

Proof. Let $\left\{a_{n}\right\} \subset A$ and $\left\{b_{n}\right\} \subset B$ be sequnces such that

$$
\lim _{n \rightarrow \infty}\left\|a_{n}-b_{n}\right\|=\operatorname{dist}(A, B)
$$

In view of reflexivity of the space $E$ and the assumption on boundedness of $A$ and $B$ we may assume (taking subsequences if necessary) that $\left\{a_{n}\right\}$ and $\left\{b_{n}\right\}$ converge weakly to points $a$ and $b$, respectively. By Mazur's theorem we deduce that $a \in A$ and $b \in B$. Next, let us observe that the sequence $\left\{a_{n}-b_{n}\right\}$ converges weakly to the point $a-b$. Hence, in view of lower semicontinuity of the norm [1] we infer that

$$
\|a-b\| \leq \liminf _{n \rightarrow \infty}\left\|a_{n}-b_{n}\right\|=\lim _{n \rightarrow \infty}\left\|a_{n}-b_{n}\right\| .
$$

This implies that $\|a-b\|=\operatorname{dist}(A, B)$.

Finally, applying Lemma 1 we complete the proof.

In order to show that the assumption on boundedness of the sets $A$ and $B$ in the above theorem is essential, consider the following example.

Example. Take the Euclidean plane $R^{2}$. Let $A, B$ be subsets of $R^{2}$ defined as follows:

$$
\begin{aligned}
& A=\{(x, 0): x \geq 0\}, \\
& B=\{(x, y): y \geq 1 / x, x>0\} .
\end{aligned}
$$

Obviously $A$ and $B$ are closed, convex but unbounded subsets of $R^{2}$. It is easily seen that the mapping $P_{A} P_{B}$ has no fixed points in the set $A$.

On the other hand dist $(A, B)=0$ but do not exist points $a \in A$ and $b \in B$ such that $\|a-b\|=0$. 


\section{References}

[1] M. M. Day, Normed Linear Spaces, Springer, Berlin-Heidelberg, New York, 1973.

[2] K. Goebel and S. Reich, Uniform Convexity, Hyperbolic Geomety, and Nonexpansive Mappings, Marcel Dekker, New York, 1984.

[3] G. Köthe, Toplogical Vector Spaces I, Springer, Berlin, 1969.

Department of Mathematics, Faculty of Science, Alexandria University, Alexandria, Egypt. 